\title{
Activation of Akt by SC79 protects myocardiocytes from oxygen and glucose deprivation (OGD)/re-oxygenation
}

\author{
Koulong Zheng ${ }^{1}$, Qing Zhang ${ }^{1}$, Gang Lin ${ }^{1}$, Yefei $\mathrm{Li}^{1}$, Zhenqiang Sheng ${ }^{1}$, Jue Wang ${ }^{1}$, \\ Liang Chen ${ }^{1}$, Hui-he Lu ${ }^{1}$ \\ ${ }^{1}$ Department of Cardiology, The Second Affiliated Hospital of Nantong University, Nantong, China \\ Correspondence to: Hui-he Lu, email: nantongluhuihe@163.com \\ Keywords: ischemic heart diseases, oxygen glucose deprivation (OGD), myocardial cells, SC79, Akt \\ Received: December 08, $2016 \quad$ Accepted: January 11, $2017 \quad$ Published: January 21, 2017
}

\section{ABSTRACT}

SC79 is a novel Akt activator. The current study tested its potential effect against oxygen and glucose deprivation (OGD)/re-oxygenation-induced myocardial cell death. We showed that SC79 activated Akt and protected H9c2 myocardial cells and primary murine myocardiocytes from OGD/re-oxygenation. Reversely, Akt inhibitor MK-2206 or Akt1 shRNA knockdown almost completely abolished SC79-mediated myocardial cytoprotection. SC79 treatment in H9c2 cells inhibited OGD/re-oxygenation-induced programmed necrosis pathway, evidenced by mitochondrial depolarization and cyclophilin D-p53-ANT-1 (adenine nucleotide translocator 1) association. Further, SC79 activated Akt downstream NF-E2-related factor 2 (NRF2) signaling to suppress OGD/re-oxygenation-induced reactive oxygen species (ROS) production. Reversely, NRF2 shRNA knockdown in H9c2 cells largely attenuated SC79-induced ROS scavenging ability and cytoprotection against OGD/ re-oxygenation. Together, we conclude that activation of Akt by SC79 protects myocardial cells from OGD/re-oxygenation.

\section{INTRODUCTION}

Ischemic heart diseases are major threat to human health, which contribute to significant human mortalities each year $[1,2]$. The incidence of these diseases, on the other hand, has been steadily rising $[1,2]$. Thus, understanding the associated pathological mechanisms and developing possible intervention strategies are vital to fight these diseases $[1,2]$. Our lab [3, 4] and others have been applying oxygen glucose deprivation (OGD) in cultured myocardial cells to mimic ischemic cell damages [5-7]. Sustained OGD ( $>1$ hour) coupling with re-oxygenation is shown to disrupt mitochondrial function, causing reactive oxygen species (ROS) production and cell necrosis (but not apoptosis) [5-7].

Recent research efforts have developed a specific, potent and cell-permeable Akt small molecule activator, named SC79 [8]. SC79 inhibits Akt membrane translocation, yet simultaneously activates Akt in the cytosol [8]. Existing studies have reported the pro-survival potential of this compound in various experimental settings [8-11]. For example, Jo et al., showed that SC79 protects neurons from stroke in vivo and in vivo. Gong et al., recently demonstrated that SC79 activates Akt signaling and protects retinal pigment epithelium cells from UV radiation [11]. Similarly, this novel Akt activator could rescue osteoblasts from dexamethasone [10]. The potential effect and the underlying signaling mechanisms of SC79 against OGD/re-oxygenation-induced myocardial cell death were tested in the current study.

NF-E2-related factor 2 (NRF2) dictates the transcription of key anti-oxidant genes via coupling with antioxidantresponsive element (ARE) in nuclei $[12,13]$. Transcription of these genes, including heme oxygenase-1 (HO1), NADPH quinone oxidoreductase 1 (NQO1) and glutamate cysteine ligase catalytic subunit (GCLC), could significantly inhibit ROS production and oxidative stress [14]. It is known that ROS production is the primary upstream mechanism to provoke the above mitochondrial necrosis pathway by $\mathrm{OGD} /$ re-oxygenation [3, 15]. Indeed, several cytoprotective agents, including salidroside and the SphK1 activator K6PC-5, were shown to inhibit OGD/re-oxygenation-induced ROS production and subsequent mitochondrial necrosis in myocardial cells $[3,15]$. In the current study, we show that SC79 protects myocardial cells from OGD/re-oxygenation via activating Akt downstream Nrf2 signaling. 


\section{RESULTS}

\section{SC79 protects myocardial cells from OGD/re- oxygenation}

To study the potential effect of SC79 on ischemic damages, H9c2 myocardial cells $[3,4]$ were maintained under oxygen glucose deprivation (OGD) for 4 hours, which were then subjected to re-oxygenation for additional 24 hours. In line with our previous findings [3, 4], OGD mimicked ischemic damages and significantly inhibited H9c2 cell survival [MTT optic density (OD) reduction, Figure 1A]. Remarkably, pre-treatment for 1 hour with the Akt activator SC79 $(10 \mu \mathrm{M})[8,9,11]$ significantly attenuated OGD/re-oxygenation-induced H9c2 survival reduction (Figure 1A). Meanwhile, results in Figure 1B demonstrated that SC79 pre-treatment also dramatically inhibited OGD/re-oxygenation-induced H9c2 cell death, the latter was tested by increased lactate dehydrogenase $(\mathrm{LDH})$ release in the conditional medium $[3,4]$.

Next, the primary murine myocardiocytes were cultured (see Method). The above OGD (4 hours)/reoxygenation (24 hours) treatment again significantly inhibited cell survival (Figure 1C), and provoked cell death (Figure 1D). Similarly, pre-treatment with SC79 again dramatically decreased OGD/re-oxygenation-induced injuries to the primary murine myocardiocytes (Figure 1C and 1D). Thus, SC79 efficiently protects myocardial cells from OGD/re-oxygenation. Notably, treatment with the SC79 by itself had no significant effect on survival and death of above myocardial cells (Figure 1A-1D).

\section{SC79-induced myocardial cytoprotection against OGD/re-oxygenation requires Akt activation}

As discussed, SC79 is a newly-developed Akt activator $[8,9,11,16]$. We thus tested Akt signaling in SC79-treated myocardial cells. Western blot assay results in Figure 2A showed that treatment with SC79 $(10 \mu \mathrm{M}, 1$ hour $)$ in $\mathrm{H} 9 \mathrm{c} 2$ cells induced significant Akt activation, which was tested by phosphorylation ("p-") of Akt at both Ser-473 and Thr-308 [11, 17-19]. MK-2206, the Akt specific inhibitor [20], almost blocked Akt activation by SC79 (Figure 2A). Significantly, SC79-mediated H9c2 cytoprotection against OGD was largely attenuated with MK-2206 co-treatment (Figure 2B and 2C). In another words, SC79 was almost ineffective against $\mathrm{OGD} / \mathrm{re}$-oxygenation in the presence of MK-2206 (Figure 2B and 2C). These results suggest that Akt activation is required for SC79-induced myocardial cytoprotection against OGD/re-oxygenation.
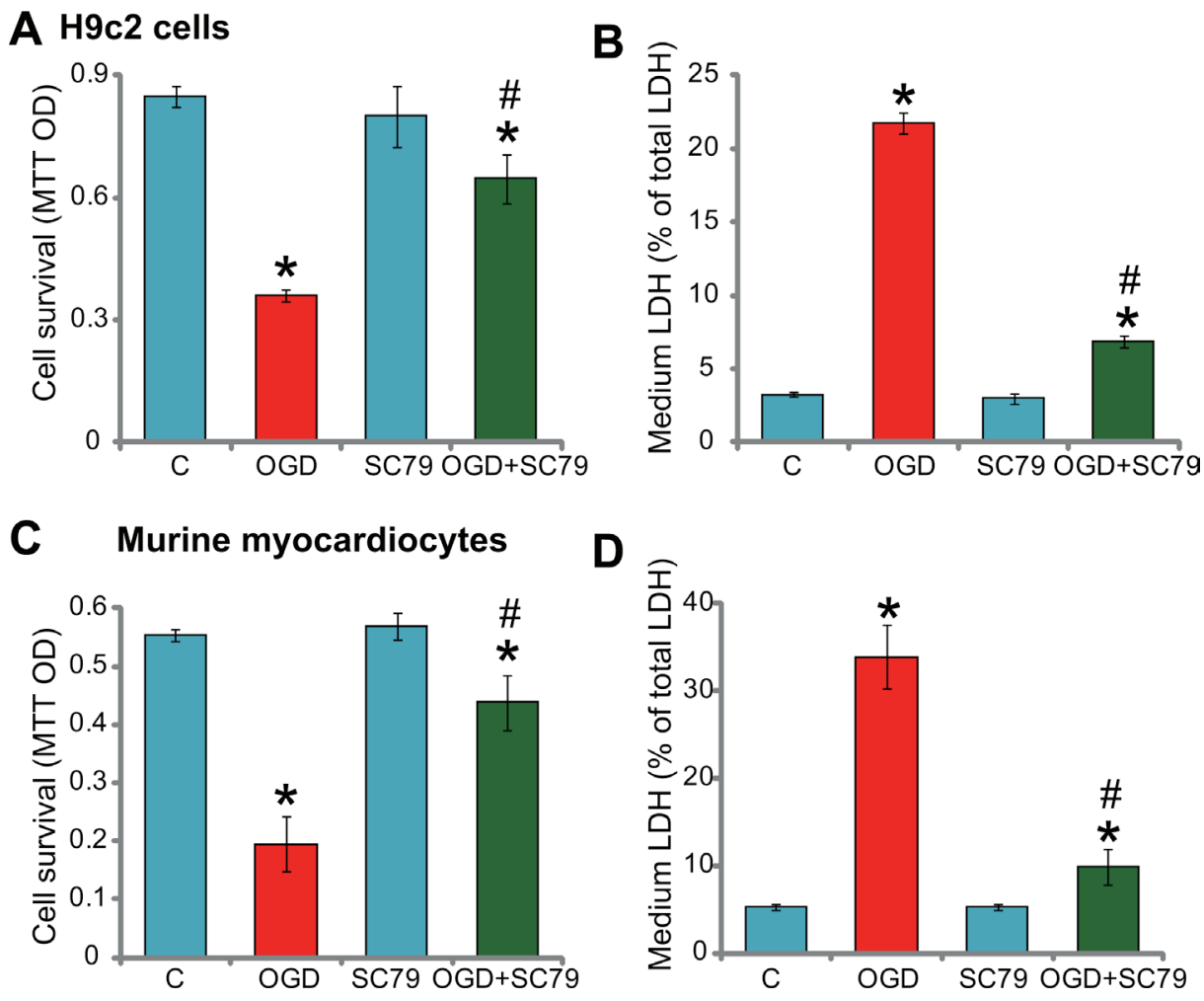

Figure 1: SC79 protects myocardial cells from OGD/re-oxygenation. H9c2 myocardial cells (A and B) or primary murine myocardiocytes (C and D), pretreated with/out SC79 (10 $\mu \mathrm{M}, 1$ hour pretreatment), were maintained under OGD for 4 hours, followed by 24 hours of re-oxygenation. Afterwards, cell survival was tested by MTT assay (A and C); Cell death was examined by the LDH release assay (B and D). "C" stands for untreated control (Same for all figures). "OGD” stands for OGD/re-oxygenation (Same for all figures). Bars indicate standard deviation ( $\mathrm{SD}, n=5)$. Each experiment was repeated five times and similar results were obtained. " $p<0.05$ vs. group "C". "p < 0.05 vs. "OGD” group. 
To further support the above hypothesis, shRNA strategy was applied to knockdown Akt1. As demonstrated, the lentiviral Akt1 shRNA led to almost complete depletion of Akt1 in H9c2 cells (Figure 2A). Consequently, SC79-provoked Akt activation was completely blocked in Akt1-silenced cells (Figure 2A). Remarkably, as shown in Figure 2B and 2C, SC79 was similarly ineffective against OGD/re-oxygenation in Akt1-silenced H9c2 cells. Significantly, the Akt specific inhibitor MK2206 almost nullified SC79-mediated cytoprotection in the primary murine myocardiocytes (Figure 2D). These shRNA results further confirmed that Akt activation is required for SC79-indued myocardial cytoprotection against OGD/re-oxygenation.

\section{OGD/re-oxygenation-induced myocardial cell death is exacerbated with Akt inhibition, but is attenuated with forced-activation of Akt}

Based on the results above, we would speculate that Akt inhibition may exacerbate OGD/re-oxygenationinduced myocardial cell death. MK-2206 and Akt1 shRNA were applied again to block Akt activation (p-Akt at Ser-473 and Thr-308) in H9c2 cells with OGD/re-oxygenation
(Figure 3A). Significantly, as shown in Figure 3B and 3C, MK-2206 and Akt1 shRNA largely intensified $\mathrm{OGD} /$ re-oxygenation-induced $\mathrm{H} 9 \mathrm{c} 2$ cell viability reduction (Figure 3B) and cell death (Figure 3C). These results indicate that basal Akt activation is also important for the survival of $\mathrm{H} 9 \mathrm{c} 2$ cells under OGD/re-oxygenation.

Next, a constitutively active Akt1 ("ca-Akt1", Flagtagged) expression vector [21] was introduced to H9c2 cells (see Method). Western blot assay results in Figure 3D confirmed that ca-Akt1 indeed dramatically provoked Akt activation (p-Akt at Ser-473 and Thr-308) in H9c2 cells. Remarkably, H9c2 cells expressing the ca-Akt1 were resistant to $\mathrm{OGD} /$ re-oxygenation (Figure $3 \mathrm{E}$ and $3 \mathrm{~F}$ ). Therefore, similar to SC79 co-treatment, forced activation of Akt by expressing ca-Akt1 also inhibited OGD/reoxygenation damages in myocardial cells.

\section{Activation of Akt by SC79 inhibits OGD/re- oxygenation-provoked programmed necrosis pathway in myocardial cells}

Previous studies have indicated that OGD/ re-oxygenation shall activate mPTP (mitochondrial permeability transition pore)-dependent necrosis
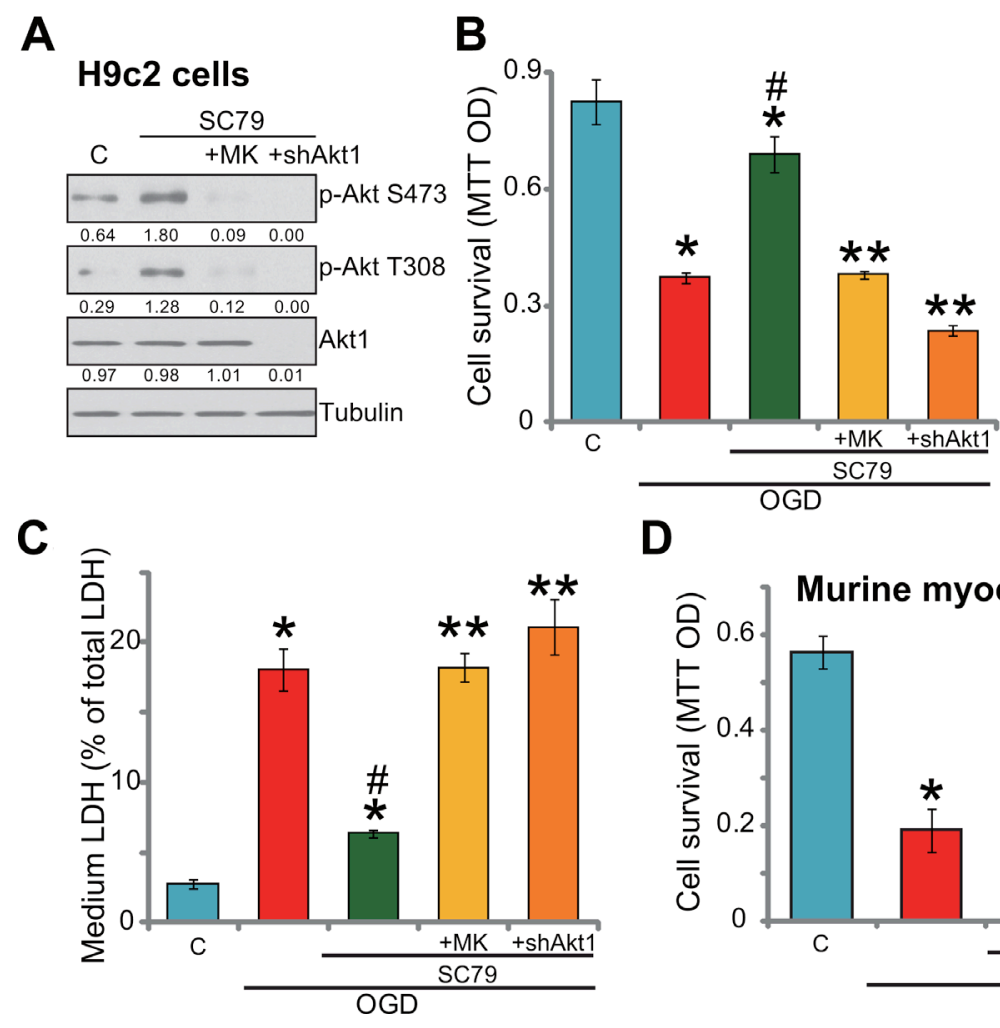

D

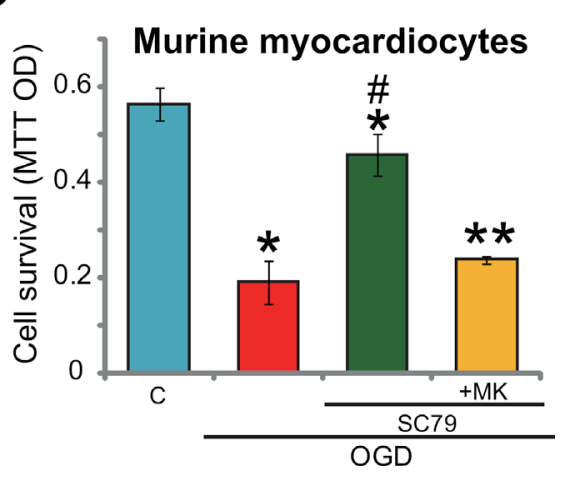

Figure 2: SC79-induced myocardial cytoprotection against OGD/re-oxygenation requires Akt activation. H9c2 myocardial cells (A-C) with/out Akt1 shRNA ("shAkt1"), or the primary murine myocardiocytes (D) were treated with SC79 (10 $\mu \mathrm{M})$ or plus MK-2206 ("MK", $10 \mu \mathrm{M}$ ) for 1 hour, expression of indicated proteins was shown (A); After the above treatment, cells were further subjected to OGD/re-oxygenation stimulation for 24 hours, cell survival (B and D, MTT assay) and cell death (C, LDH release assay) were tested. Akt1 expression (vs. Tubulin) and Akt phosphorylation (vs. Tubulin) were quantified. Bars indicate standard deviation (SD, $n=5$ ). Each experiment was repeated three times and similar results were obtained. ${ }^{*} p<0.05$ vs. "C" group. ${ }^{*} p<0.05$ vs. "OGD" group. ${ }^{* *} p<0.05$ vs. "SC79" group. 
pathway (but not apoptosis) to mediate myocardial cell death $[3,15]$. This mitochondria-mediated cell necrosis pathway is also named programmed necrosis [22-25]. In this study, treatment of OGD/re-oxygenation in the H9c2 cells apparently also provoked this programmed-necrosis pathway, which was evidenced by reactive oxygen species (ROS) production (4A), mitochondrial association of p53-cyclophilin D (Cyp-D)-ANT-1 (adenine nucleotide translocator 1) [26] (Figure 4B) as well as mitochondrial depolarization (Figure 4C). Remarkably, pre-treatment of SC79 as well as expression of ca-Akt1 largely inhibited OGD/re-oxygenation-induced above changes (Figure 4A-4C). On the other hand, blockage of basal Akt activation by MK-2206 or Akt1 shRNA aggravated OGD/ re-oxygenation-induced ROS production (Figure 4D) and mitochondrial depolarization (Figure 4E). In the primary murine myocardiocytes, pre-treatment of SC79 similarly inhibited OGD/re-oxygenation-induced ROS production (Figure 4F), mitochondrial p53-Cyp-D-ANT-1 association (Figure 4G) and depolarization (Figure 4H). These results together suggest that activation of Akt by SC79 could inhibit OGD/re-oxygenation-induced programmed necrosis pathway to possibly shut down subsequent myocardial cell death.

\section{SC79 activates Akt downstream NRF2 signaling in myocardial cells}

Recent studies have shown that SC79 could activate NRF2 signaling to inhibit oxidative damages [10, 11]. Here, we found that SC79 treatment in H9c2 cells also induced significant mRNA expression of NRF2-regualted genes, including $\mathrm{HO}$, NQO1 and GCLC (Figure 5A-5C) $[10,11,27,28]$. Notably, the Akt inhibitor MK-2206 and Akt1 shRNA almost blocked SC79-induced above gene expression (Figure 5A-5C), indicating that $\mathrm{Akt}$ serves as the upstream signaling for NRF2 activation by SC79. Notably, exogenous expression of ca-Akt1 in $\mathrm{H} 9 \mathrm{c} 2$ cells also induced mRNA expression of above Nrf2 genes (HO1, NQO1 and GCLC, Figure 5D). To confirm that the expression of these mRNAs was through NRF2, shRNA strategy was again applied to silence NRF2. As

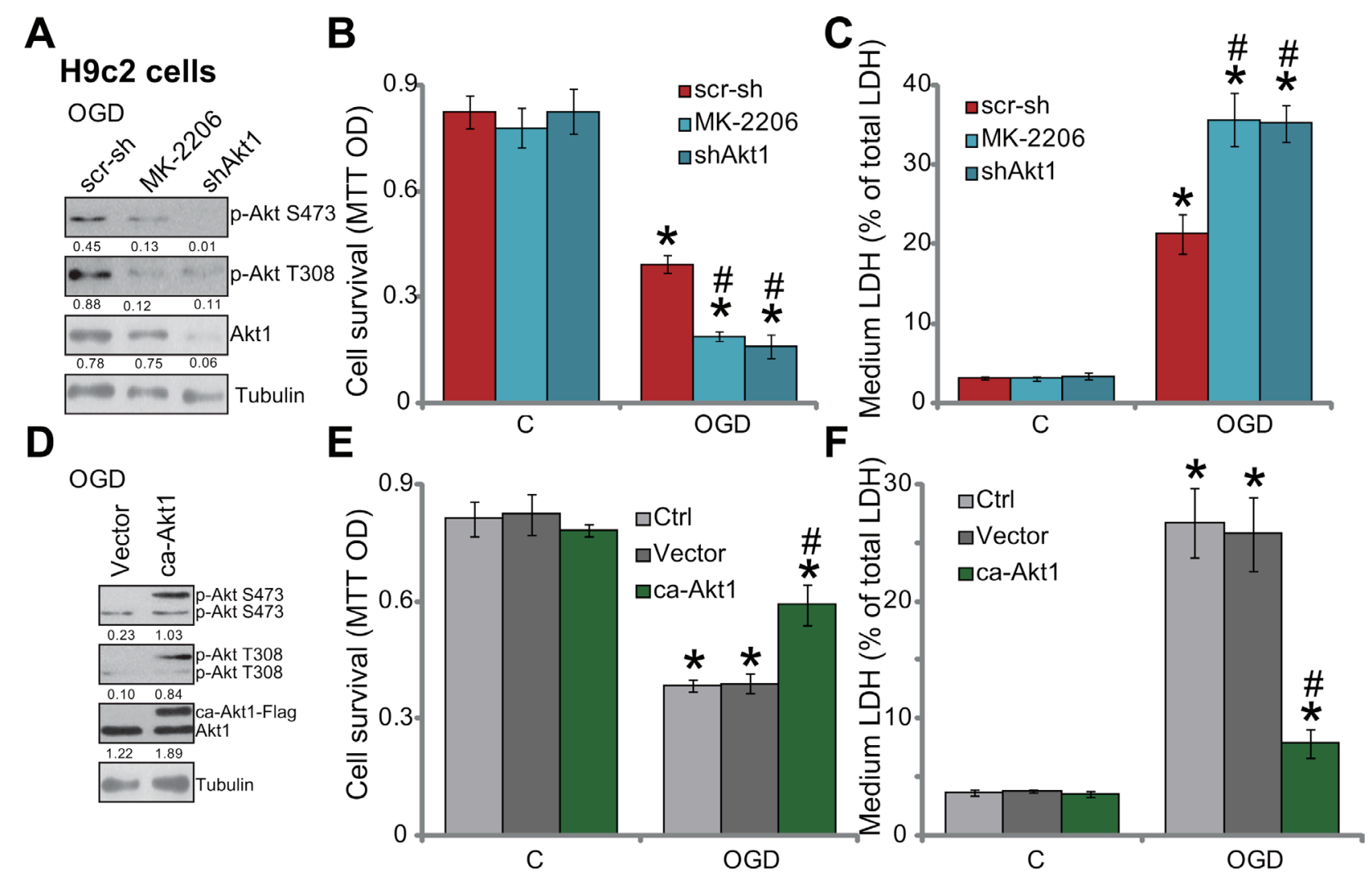

Figure 3: OGD/re-oxygenation-induced myocardial cell death is exacerbated with Akt inhibition, but is attenuated with forced-activation of Akt. H9c2 myocardial cells, expressing Akt1 shRNA ("shAkt1") or scramble shRNA ("scr-sh"), were treated with/out MK-2206 (10 $\mu \mathrm{M})$, cells were further subjected to OGD/re-oxygenation; Expression of indicated proteins was shown (A, 3 hours after re-oxygenation); Cell survival (B, 24 hours after re-oxygenation) and cell death (C, 24 hours after re-oxygenation) were also tested. H9c2 cells, expressing constitutively active Akt1 ("ca-Akt1", Flag-tagged) or empty vector (pSuper-puro, "Vector"), were treated with/out OGD/re-oxygenation; Expression of indicated proteins was shown (D, 3 hours after re-oxygenation); Cell survival (E, 24 hours after reoxygenation) and cell death (F, 24 hours after re-oxygenation) were tested as well. Akt1 expression (vs. Tubulin) and Akt phosphorylation (vs. Tubulin) were quantified (A and D). Bars indicate standard deviation $(\mathrm{SD}, n=4)$. Each experiment was repeated three times and similar results were obtained. " $p<0.05$ vs. "C" group. $\# p<0.05$ vs. "scr-sh"/“Vector" group. 
demonstrated, the two applied shRNAs (see our previous study [3]) dramatically downregulated NRF2 expression in H9c2 cells (Figure 5F). Consequently, SC79-induced mRNA and protein expression of above Nrf2 genes were almost blocked (Figure 5E-5F). These results imply that SC79 activates Akt-dependent NRF2 signaling in H9c2 cells. Significantly, SC79-mediated anti-oxidant activity in OGD/re-oxygenation-treated cells was almost nullified with NRF2 shRNA knockdown (Figure 5G). Further, in the NRF2-silenced cells, SC79-indced cytoprotection against OGD/re-oxygenation was also obviously compromised (Figure 5H and 5I). Therefore, activation of

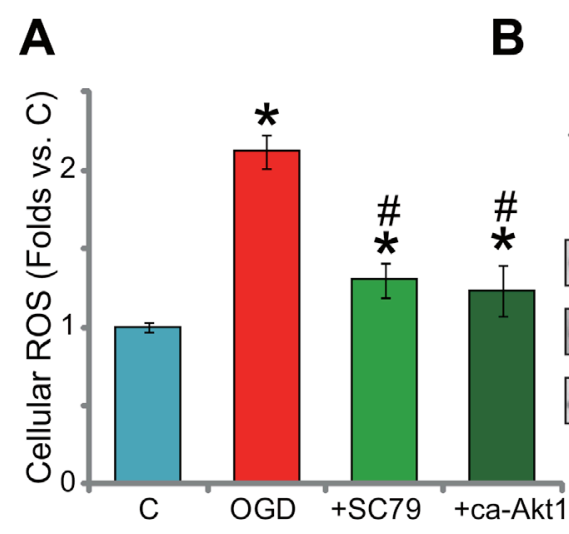

D
Mito-IP: Cyp-D Mito-IB (Input)

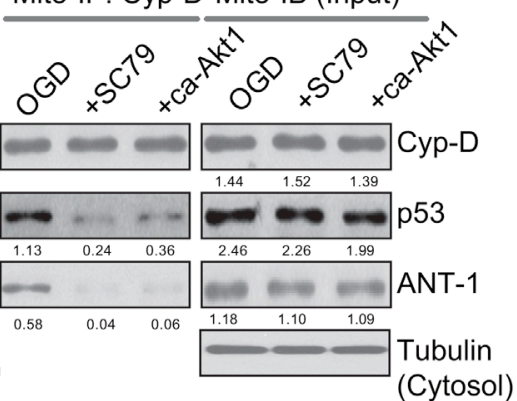

(Cytosol)
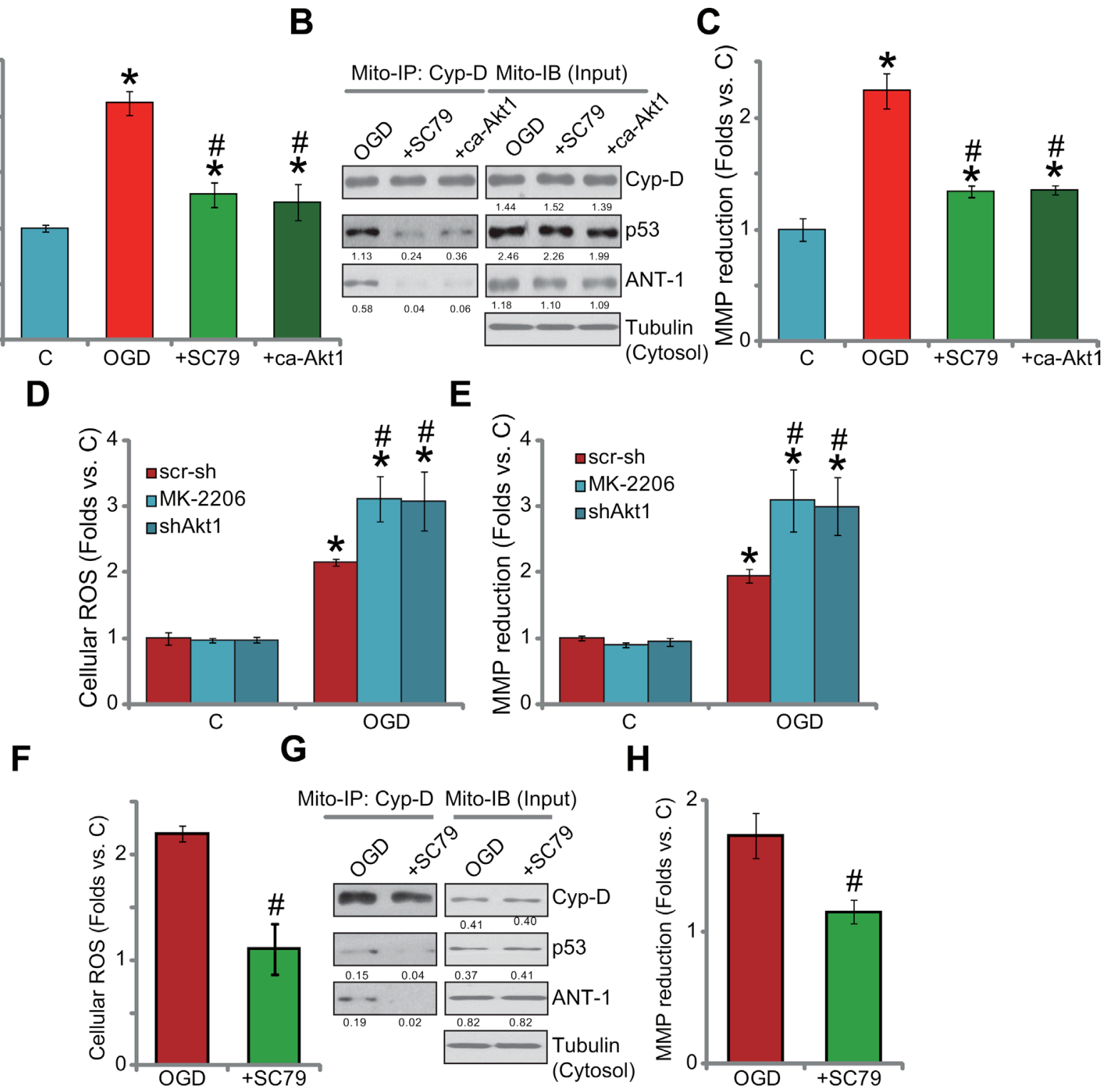

Mito-IP: Cyp-D Mito-IB (Input)
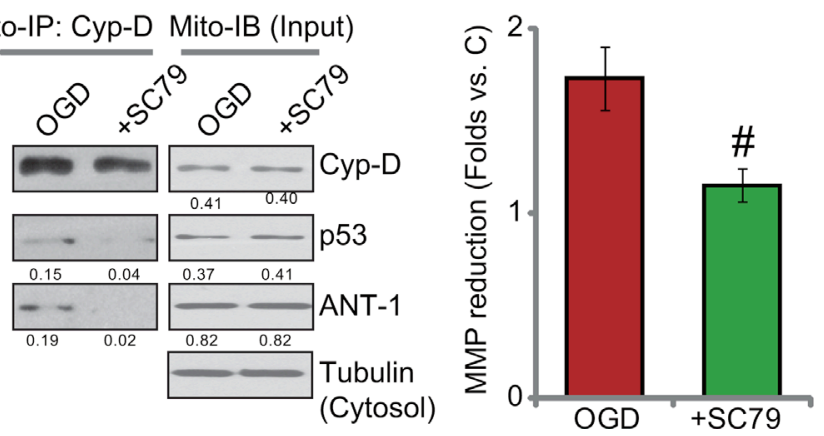

Figure 4: Activation of Akt by SC79 inhibits OGD/re-oxygenation-provoked programmed necrosis pathway in myocardial cells. H9c2 cells, with/out constitutively active Akt1 ("ca-Akt1", Flag-tagged), were treated with OGD/re-oxygenation or plus SC79 $(10 \mu \mathrm{M}, 1$ hour pre-treatment); ROS production was tested 4 hours after re-oxygenation (A); After 6 hours, the complexation and expression of listed proteins in the mitochondrial lysates were tested by mitochondrial immunoprecipitation assay ("Mito-IP" of Cyp-D) and mitochondrial immuno-blot assay ("Mito-IB", as "Input"), respectively (B); The mitochondrial membrane potential (MMP) reduction (JC10 assay, C) was also tested (6 hours after re-oxygenation). The effect of MK-2206 (10 $\mu \mathrm{M}, 1$ hour pre-treatment) or lentiviral shRNA Akt1 on OGD/re-oxygenation-induced ROS production (D, after 4 hours) and mitochondrial depolarization (E, after 6 hours) was also tested. Primary murine myocardiocytes were treated with OGD/re-oxygenation or plus SC79 $(10 \mu \mathrm{M}, 1$ hour pre-treatment), ROS production (F, after 4 hours), mitochondrial p53-Cyp-D-ANT-1 complexation and expression (E, after 6 hours) and mitochondrial depolarization (H, after 6 hours) were tested using the above methods. For Mito-IP assay, the amount of Cyp-D-bound p53 or ANT-1 was quantified (vs. Cyp-D, B and G). For Mito-IB assay, expression of p53/Cyp/D-ANT-1 was quantified (vs. cytosol Tubulin, B and G). Bars indicate standard deviation $(\mathrm{SD}, n=4)$. Each experiment was repeated three times and similar results were obtained. " $p<0.05$ vs. group "C". ${ }^{\#} p<0.05$ vs. "OGD” only. 
NRF2 by SC79, as a key downstream of Akt, is required for $\mathrm{H} 9 \mathrm{c} 2$ cytoprotection against $\mathrm{OGD} /$ re-oxygenation.

\section{DISCUSSION}

A number of recent literatures proposed that activation of mitochondrial (programmed) necrosis pathway, but not apoptosis, mediates cell death by sustained OGD/re-oxygenation [3, 15, 29-32]. Following OGD/reoxygenation, intracellular ROS will be produced, which dictates cytoplasmic p53 translocation to the mitochondria, where it forms a complex with local proteins Cyp-D and ANT-1 [3, 30-32]. The Cyp-D-p53-ANT-1 association is required for subsequent $\mathrm{mPTP}$ opening, mitochondrial depolarization and cell necrosis (but not apoptosis) $[3,15$, 29-32]. Here, we found that SC79 or ca-Akt1 significantly inhibited $\mathrm{OGD} / \mathrm{re}$-oxygenation-induced programmed necrosis pathway, the latter was evidenced by ROS production, mitochondrial depolarization and Cyp-D-p53ANT-1 mitochondrial association. Thus, we here proposed a novel mechanism of SC79-mediate cytoprotection: To shut down the programmed necrosis pathway.

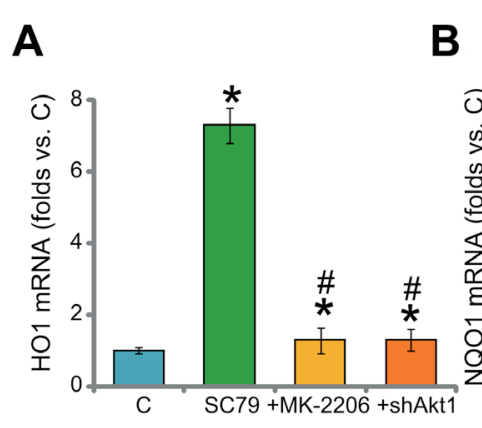

E

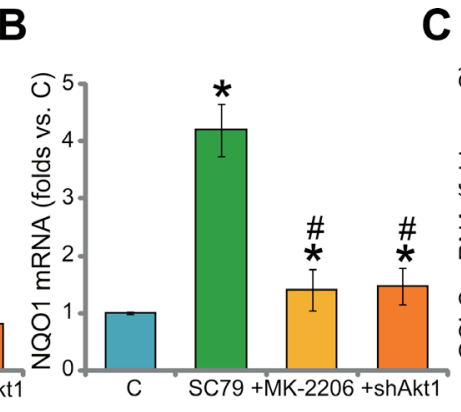

$\mathbf{F}$
C

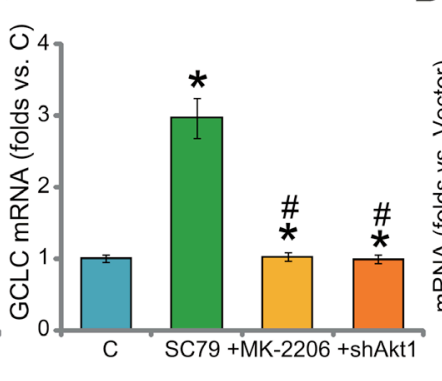

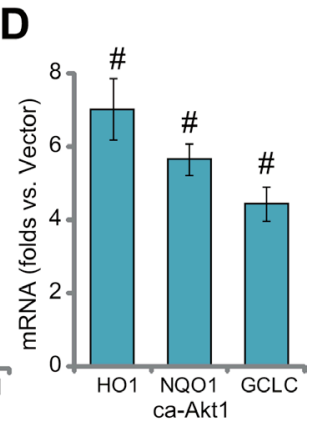

G
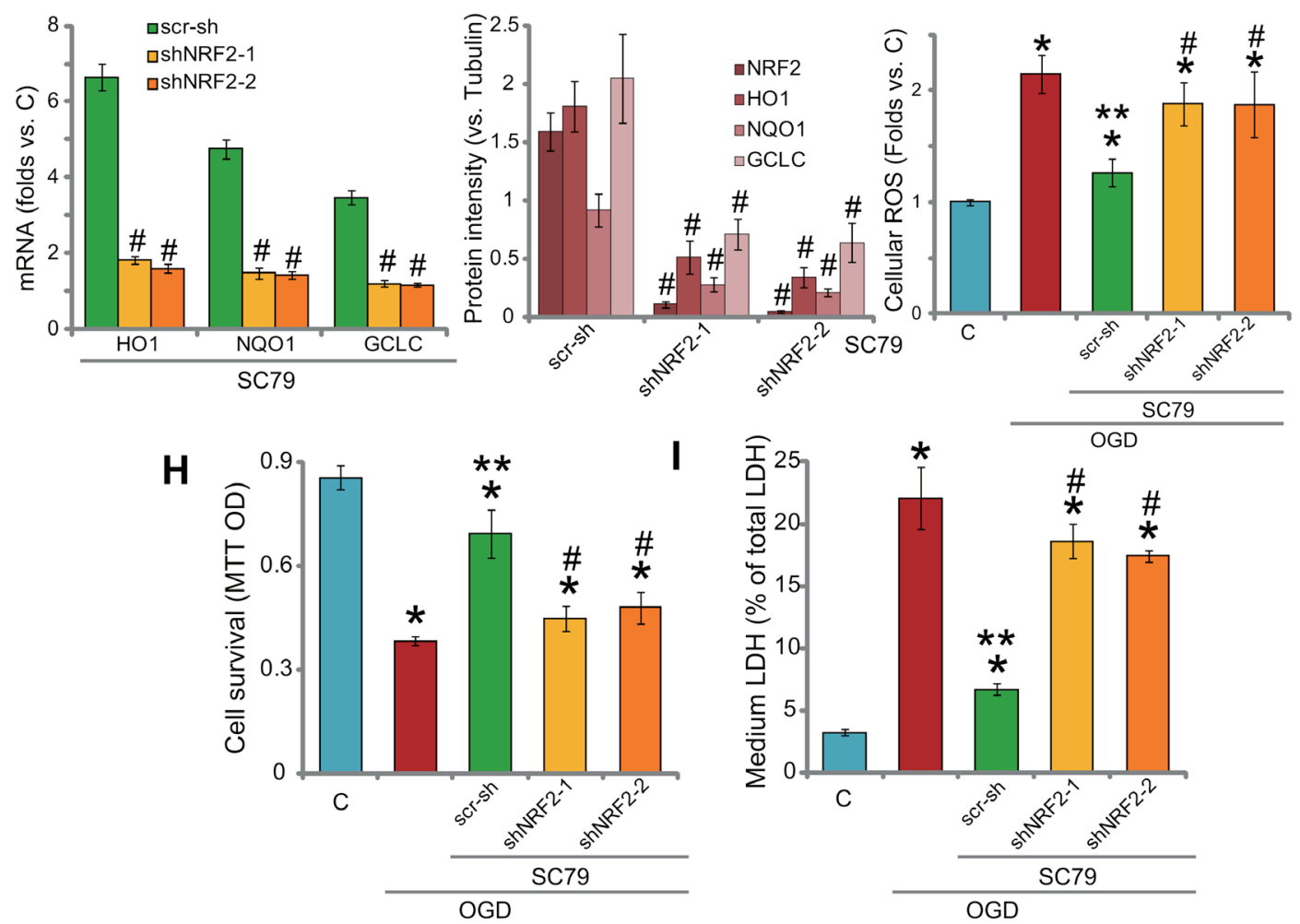

Figure 5: SC79 activates Akt downstream NRF2 signaling in myocardial cells. H9c2 myocardial cells, with Akt1 shRNA ("shAkt1"), constitutively active Akt1 (“ca-Akt1"), were treated with/out SC79 (10 $\mu$ M) or plus MK-2206 ( $\mu$ M) for 2 hours, expression of listed NRF2-regulaed mRNAs was tested by RT-PCR assay (A-D); H9c2 cells, expressing NRF2 shRNA-1/-2 ("shNRF2-1/2") or scramble control shRNA ("scr-sh"), were treated with SC79 $(10 \mu \mathrm{M})$ for 2 hours, mRNA (E) and protein (F, three repeat data were quantified, vs. Tubulin) expression of listed genes were tested; Above cells were also subjected to OGD/re-oxygenation assay; ROS production (G, 4 hours after re-oxygenation), cell survival (H, MTT assay, 24 hours after re-oxygenation) and cell death (I, LDH release assay, 24 hours after re-oxygenation) were shown. Bars indicate standard deviation $(\mathrm{SD}, n=3)$. Each experiment was repeated four times and similar results were obtained. " $p<0.05$ vs. "C" group. " $\mathrm{p}<0.05$ vs. "SC79" group (A-C). ${ }^{\#} p<0.05$ vs. "Vector" cells (D). " $p<0.05$ vs. "scr-sh" group (D-F). ${ }^{* *} p<0.05$ vs. OGD only group (G-I). 
In the present study, SC79 treatment in myocardial cells also activated NRF2 signaling, as a key downstream of Akt, to block OGD/re-oxygenation-induced ROS production. Reversely, NRF2 shRNA knockdown significantly attenuated SC79-induced ROS scavenging and cytoprotective activities. Thus, activation of NRF2 signaling by SC79 is required for myocardial cytoprotection against OGD/re-oxygenation.

Our results showing SC79 activates NRF2 signaling were consistent with recent findings $[10,11]$. Indeed, it has been proposed that Akt and its downstream mTOR complex 1 (mTORC1) could directly activate NRF2 $[33,34]$. Lee et al., found that sulforaphane provokes NRF2 signaling via PI3K-Akt [35]. Similarly, Xu et al., showed that pyocyanin-induced NRF2 activation requires activation of PI3K-Akt [36]. Salvianolic acid A and 3H-1,2-dithiole-3-thione (D3T)-activated NRF2-HO-1 signaling is also the downstream of Akt-mTORC1 signaling [33, 34]. For the mechanism study, it was shown that Akt may induce NRF2 phosphorylation at Ser-40 $[10,11,33]$, which is required for subsequent NRF2 stabilization, accumulation and nuclear translocation [33, 37, 38]. Together, we suggest that SC79 activates NRF2 as a downstream of Akt to fight against OGD/re-oxygenationinduced oxidative stress and myocardial cell death. The detailed mechanisms may warrant further studies.

\section{MATERIALS AND METHODS}

\section{Chemical and reagents}

SC79 and MK-2206 were purchased from Selleck (Beijing, China). Antibodies of cyclophilin D (Cyp-D), adenine nucleotide translocator 1 (ANT-1) and p53 as well as NRF2, heme oxygenase-1 (HO1). NADPH quinone oxidoreductase 1 (NQO1) and glutamate cysteine ligase catalytic subunit (GCLC) were obtained from Santa Cruz Biotechnology (Santa Cruz, CA). Antibodies for Akt, phosphorylated (p-) Akt (Thr-308 and Ser-473) and ( $\beta$-) tubulin were purchased from Cellular Signaling Tech (Beverly, MA).

\section{H9c2 cell culture}

As reported $[3,39,40]$, the rat embryonic ventricular $\mathrm{H} 9 \mathrm{c} 2$ myocardial cells were cultured in DMEM medium with $10 \%$ FBS.

\section{Primary culture of murine myocardiocytes}

Primary culture of murine myocardiocytes was described previously [15]. Briefly, ventricles of C57BL6 mice (at day-1) were minced and digested in $0.5 \mathrm{mg} / \mathrm{mL}$ collagenase I (Sigma) for $40 \mathrm{~min}$. The cell suspensions containing primary myocardiocytes were filtered. Cells were then cultured in M-199 medium supplemented with $10 \% \mathrm{FBS}$, and plated for $30 \mathrm{~min}$ to separate from nonmyocardiocytes. The myocardiocytes were then plated in
M-199 supplemented with 10\% FBS. A confluent monolayer with primary spontaneously beating cells was formed [15]. The protocol of culture of primary cells was approved by the Ethics Committee and IACUC of authors' institutions.

\section{OGD/re-oxygenation}

The detailed protocol of OGD/re-oxygenation was described previously [3]. Briefly, cells were cultured in a pre-warmed glucose-free balanced salt solution [3]. The solution was then bubbled with an anaerobic gas mix $(95 \%$ $\mathrm{N}_{2}, 5 \% \mathrm{CO}_{2}$ ). Cells were then incubated in the solution for 4 hours to produce oxygen and OGD and then reoxygenated for 3-24 hours.

\section{MTT cell survival assay}

Cell survival was tested by the 3-[4,5-dimethylthylthiazol-2-yl]-2,5 diphenyltetrazolium bromide (MTT) (Sigma, St. Louis, MO) assay [3, 40].

\section{LDH detection}

Following the applied treatment, cell death was detected by lactate dehydrogenase (LDH) assay using a commercial available LDH kit (Takara, Tokyo, Japan). LDH release $(\times 100 \%)$ was calculated as follows: $\mathrm{LDH}$ in conditional medium/(LDH in conditional medium + LDH in cell lysates).

\section{Western blots}

Western blot assay was performed as described previously [3, 40]. Band intensity was quantified and normalized to loading control.

\section{Mitochondrial immunoprecipitation (Mito-IP)}

For each treatment, mitochondria of 10 million $\mathrm{H} 9 \mathrm{c} 2$ cells were obtained via "Mitochondria Isolation Kit for Cultured Cells" from Thermo Scientific (Hudson, NH), which was then lysed [3]. Immunoprecipitation (IP) was performed via the anti-Cyp-D antibody (see [30]), and immune complexes were captured with protein G-Sepharose. Afterward, IP samples were subjected to Western blot assay, p53-Cyp-D-ANT-1 association was detected $[3,30]$.

\section{Real-time PCR}

RNA extraction and reverse transcription were performed as described previously [3] . Real-time PCR was performed on a Bio-Rad IQ5 multicolor detection system. After amplification, melt curve analysis was performed to analyze product melting temperature. GAPDH was tested as internal control, and $2^{-\Delta \triangle \mathrm{CT}}$ method [41] was applied to quantify mRNA expression. The primers for rat $\mathrm{HO}-1$, GAPDH NQO-1 and GCLC were described previously [3]. 
The primers for murine HO-1, GAPDH, GCLC, NQO-1 were from Dr. Jiang's group [33, 34].

\section{ROS detection}

Following treatment of cells, ROS production was determined by carboxy-H2DCFDA dye assay [3]. The ROS intensity was recorded on a spectrofluorometer using excitation and emission filters of 488 and $530 \mathrm{~nm}$, respectively [3].

\section{Detection of mitochondrial membrane potential (MMP)}

MMP was measured through JC-10 dye (Invitrogen) to reflect mitochondrial depolarization [42]. Briefly, after indicated treatment, cells were stained with JC-10 $(5.0 \mu \mathrm{g} / \mathrm{mL})$, which were then washed, tested immediately on a spectrofluorometer.

\section{NRF2 knockdown by shRNA}

The two non-overlapping lentiviral NRF2 shRNAs were described previously [3]. The lentiviral Akt1 (rat) shRNA was purchased from Santa Cruz Biotech. The lentiviral shRNA $(20 \mu \mathrm{L} / \mathrm{mL})$ was added to cells for 12 hours, and cells were selected by puromycin $(0.25 \mu \mathrm{g} / \mathrm{mL})$ for another 24 hours. NRF2 or Akt1 expression in the selected cells was tested by Western blot assay. Control cells were transfected with same amount of scramble control shRNA (Santa Cruz, Shanghai, China).

\section{Constitutively active Akt1 expression}

The construct with constitutively active mutant of Akt1 ("ca-Akt1", Flag-tagged) as well as empty vector (pSuper-puro) were provided by Jiang's group [21]. The construct was transfected to $\mathrm{H} 9 \mathrm{c} 2$ cells via Lipofectamine 2000 (Invitrogen). Cells were then subjected to puromycin $(0.25 \mu \mathrm{g} / \mathrm{mL})$ selection for 48 hours. Expression of Akt1 in the selected cells was tested by Western blot assay.

\section{Statistical analysis}

Statistical significance was determined by one-way ANOVA by Dunnett's test as reported [3, 4, 34].

\section{CONCLUSIONS}

Activation of Akt by SC79 protects myocardial cells from OGD/re-oxygenation damages.

\section{ACKNOWLEDGMENTS}

This work is supported by the Science Foundation of Nantong City (MS22015006 to K.Z.).

\section{CONFLICTS OF INTEREST}

The listed authors have no conflicts of interests.

\section{Authors' contributions}

All authors carried out the experiments, participated in the design of the study and performed the statistical analysis, participated in its design and coordination and helped to draft the manuscript.

\section{REFERENCES}

1. Nabel EG, Braunwald E. A tale of coronary artery disease and myocardial infarction. N Engl J Med. 2012; 366:54-63.

2. Steptoe A, Kivimaki M. Stress and cardiovascular disease. Nat Rev Cardiol. 2012; 9:360-370.

3. Zheng K, Sheng Z, Li Y, Lu H. Salidroside inhibits oxygen glucose deprivation (OGD)/re-oxygenation-induced H9c2 cell necrosis through activating of Akt-Nrf2 signaling. Biochem Biophys Res Commun. 2014; 451:79-85.

4. Zheng K, Lu H, Sheng Z, Li Y, Xu B. Low-concentration of perifosine surprisingly protects cardiomyocytes from oxygen glucose deprivation. Biochem Biophys Res Commun. 2015.

5. Ekhterae D, Lin Z, Lundberg MS, Crow MT, Brosius FC, 3rd, Nunez G. ARC inhibits cytochrome c release from mitochondria and protects against hypoxia-induced apoptosis in heart-derived H9c2 cells. Circ Res. 1999; 85:e70-77.

6. Marambio P, Toro B, Sanhueza C, Troncoso R, Parra V, Verdejo H, Garcia L, Quiroga C, Munafo D, Diaz-Elizondo J, Bravo R, Gonzalez MJ, Diaz-Araya G, et al. Glucose deprivation causes oxidative stress and stimulates aggresome formation and autophagy in cultured cardiac myocytes. Biochim Biophys Acta. 2010; 1802:509-518.

7. Persky AM, Green PS, Stubley L, Howell CO, Zaulyanov L, Brazeau GA, Simpkins JW. Protective effect of estrogens against oxidative damage to heart and skeletal muscle in vivo and in vitro. Proc Soc Exp Biol Med. 2000; 223:59-66.

8. Jo H, Mondal S, Tan D, Nagata E, Takizawa S, Sharma AK, Hou Q, Shanmugasundaram K, Prasad A, Tung JK, Tejeda AO, Man H, Rigby AC, et al. Small molecule-induced cytosolic activation of protein kinase Akt rescues ischemia-elicited neuronal death. Proc Natl Acad Sci USA. 2012; 109:10581-10586.

9. Zhang D, Zhang H, Hao S, Yan H, Zhang Z, Hu Y, Zhuang Z, Li W, Zhou M, Li K, Hang C. Akt Specific Activator SC79 Protects against Early Brain Injury following Subarachnoid Hemorrhage. ACS Chem Neurosci. 2016.

10. Li ST, Chen NN, Qiao YB, Zhu WL, Ruan JW, Zhou XZ. SC79 rescues osteoblasts from dexamethasone though activating Akt-Nrf2 signaling. Biochem Biophys Res Commun. 2016; 479:54-60.

11. Gong YQ, Huang W, Li KR, Liu YY, Cao GF, Cao C, Jiang Q. SC79 protects retinal pigment epithelium cells 
from UV radiation via activating Akt-Nrf2 signaling. Oncotarget. 2016; 7:60123-60132. doi: 10.18632/ oncotarget.11164.

12. Suzuki T, Yamamoto M. Molecular basis of the Keap1-Nrf2 system. Free Radic Biol Med. 2015; 88:93-100.

13. Li W, Kong AN. Molecular mechanisms of Nrf2-mediated antioxidant response. Mol Carcinog. 2009; 48:91-104.

14. Sporn MB, Liby KT. NRF2 and cancer: the good, the bad and the importance of context. Nat Rev Cancer. 2012; 12:564-571.

15. Shao JJ, Peng Y, Wang LM, Wang JK, Chen X. Activation of SphK1 by K6PC-5 Inhibits Oxygen-Glucose Deprivation/ Reoxygenation-Induced Myocardial Cell Death. DNA Cell Biol. 2015; 34:669-676.

16. Moreira JB, Wohlwend M, Alves MN, Wisloff U, Bye A. A small molecule activator of AKT does not reduce ischemic injury of the rat heart. J Trans1 Med. 2015; 13:76.

17. Vivanco I, Sawyers CL. The phosphatidylinositol 3-Kinase AKT pathway in human cancer. Nat Rev Cancer. 2002; 2:489-501.

18. Hennessy BT, Smith DL, Ram PT, Lu Y, Mills GB. Exploiting the PI3K/AKT pathway for cancer drug discovery. Nat Rev Drug Discov. 2005; 4:988-1004.

19. Cao C, Huang X, Han Y, Wan Y, Birnbaumer L, Feng GS, Marshall J, Jiang M, Chu WM. Galpha(i1) and Galpha(i3) are required for epidermal growth factor-mediated activation of the Akt-mTORC1 pathway. Sci Signal. 2009; 2:ra17.

20. Hirai H, Sootome H, Nakatsuru Y, Miyama K, Taguchi S, Tsujioka K, Ueno Y, Hatch H, Majumder PK, Pan BS, Kotani H. MK-2206, an allosteric Akt inhibitor, enhances antitumor efficacy by standard chemotherapeutic agents or molecular targeted drugs in vitro and in vivo. Mol Cancer Ther. 2010; 9:1956-1967.

21. Jiang F, Jin K, Huang S, Bao Q, Shao Z, Hu X, Ye J. Liposomal C6 Ceramide Activates Protein Phosphatase 1 to Inhibit Melanoma Cells. PLoS One. 2016; 11:e0159849.

22. Zhang LY, Wu YL, Gao XH, Guo F. Mitochondrial protein cyclophilin-D-mediated programmed necrosis attributes to berberine-induced cytotoxicity in cultured prostate cancer cells. Biochem Biophys Res Commun. 2014; 450:697-703.

23. Qin LS, Jia PF, Zhang ZQ, Zhang SM. ROS-p53cyclophilin-D signaling mediates salinomycin-induced glioma cell necrosis. J Exp Clin Cancer Res. 2015; 34:57.

24. Ouyang L, Shi Z, Zhao S, Wang FT, Zhou TT, Liu B, Bao JK. Programmed cell death pathways in cancer: a review of apoptosis, autophagy and programmed necrosis. Cell Prolif. 2012; 45:487-498.

25. Ju T, Gao D, Fang ZY. Targeting colorectal cancer cells by a novel sphingosine kinase 1 inhibitor PF-543. Biochem Biophys Res Commun. 2016; 470:728-734.

26. Baines CP, Kaiser RA, Purcell NH, Blair NS, Osinska H, Hambleton MA, Brunskill EW, Sayen MR, Gottlieb RA, Dorn GW, Robbins J, Molkentin JD. Loss of cyclophilin D reveals a critical role for mitochondrial permeability transition in cell death. Nature. 2005; 434:658-662.
27. Zhang Q, Pi J, Woods CG, Andersen ME. A systems biology perspective on Nrf2-mediated antioxidant response. Toxicol Appl Pharmacol. 2010; 244:84-97.

28. Zhang H, Davies KJ, Forman HJ. Oxidative stress response and Nrf2 signaling in aging. Free Radic Biol Med. 2015; 88:314-336.

29. Zhao LP, Ji C, Lu PH, Li C, Xu B, Gao H. Oxygen glucose deprivation (OGD)/re-oxygenation-induced in vitro neuronal cell death involves mitochondrial cyclophilin-D/ P53 signaling axis. Neurochem Res. 2013; 38:705-713.

30. Vaseva AV, Marchenko ND, Ji K, Tsirka SE, Holzmann S, Moll UM. p53 opens the mitochondrial permeability transition pore to trigger necrosis. Cell. 2012; 149:1536-1548.

31. Karch J, Molkentin JD. Is p53 the Long-Sought Molecular Trigger for Cyclophilin D-Regulated Mitochondrial Permeability Transition Pore Formation and Necrosis? Circ Res. 2012; 111:1258-1260.

32. Baumann K. Cell death: multitasking p53 promotes necrosis. Nat Rev Mol Cell Biol. 2012; 13:480-481.

33. Li KR, Yang SQ, Gong YQ, Yang H, Li XM, Zhao YX, Yao J, Jiang Q, Cao C. 3H-1,2-dithiole-3-thione protects retinal pigment epithelium cells against Ultra-violet radiation via activation of Akt-mTORC1-dependent Nrf2-HO-1 signaling. Sci Rep. 2016; 6:25525.

34. Zhang H, Liu YY, Jiang Q, Li KR, Zhao YX, Cao C, Yao J. Salvianolic acid A protects RPE cells against oxidative stress through activation of Nrf2/HO-1 signaling. Free Radic Biol Med. 2014; 69:219-228.

35. Lee YJ, Jeong HY, Kim YB, Won SY, Shim JH, Cho MK, Nam HS, Lee SH. Reactive oxygen species and PI3K/Akt signaling play key roles in the induction of Nrf2-driven heme oxygenase- 1 expression in sulforaphane-treated human mesothelioma MSTO-211H cells. Food Chem Toxicol. 2012; 50:116-123.

36. Xu Y, Duan C, Kuang Z, Hao Y, Jeffries JL, Lau GW. Pseudomonas aeruginosa Pyocyanin Activates NRF2-AREMediated Transcriptional Response via the ROS-EGFRPI3K-AKT/MEK-ERK MAP Kinase Signaling in Pulmonary Epithelial Cells. PLoS One. 2013; 8:e72528.

37. Huang HC, Nguyen T, Pickett CB. Phosphorylation of $\mathrm{Nrf} 2$ at Ser-40 by protein kinase $\mathrm{C}$ regulates antioxidant response element-mediated transcription. J Biol Chem. 2002; 277:42769-42774.

38. Gu DM, Lu PH, Zhang K, Wang X, Sun M, Chen GQ, Wang Q. EGFR mediates astragaloside IV-induced Nrf2 activation to protect cortical neurons against in vitro ischemia/reperfusion damages. Biochem Biophys Res Commun. 2015; 457:391-397.

39. Chen MB, Wu XY, Gu JH, Guo QT, Shen WX, Lu PH. Activation of AMP-activated protein kinase contributes to doxorubicin-induced cell death and apoptosis in cultured myocardial H9c2 cells. Cell Biochem Biophys. 2011; 60:311-322.

40. Chen Z, Shen X, Shen F, Zhong W, Wu H, Liu S, Lai J. TAK1 activates AMPK-dependent cell death pathway in 
hydrogen peroxide-treated cardiomyocytes, inhibited by heat shock protein-70. Mol Cell Biochem. 2013; 377:35-44.

41. Livak KJ, Schmittgen TD. Analysis of relative gene expression data using real-time quantitative PCR and the 2(-Delta Delta C(T)) Method. Methods. 2001; 25:402-408.
42. Zhen YF, Wang GD, Zhu LQ, Tan SP, Zhang FY, Zhou XZ, Wang XD. P53 Dependent Mitochondrial Permeability Transition Pore Opening Is Required for DexamethasoneInduced Death of Osteoblasts. J Cell Physiol. 2014. 